\title{
Gonadotropin-releasing Hormone Agonist Down Regulation Combined With Hormone Replacement Therapy Improves the Reproductive Outcome in the Frozen-thawed Embryo Transfer Cycle of Elderly Patients With Idiopathic Recurrent Implantation Failure
}

\section{Dan Pan}

Xi'an Jiaotong University affiliated Northwest Women and Children's Hospital: Northwest Women and Children's Hospital

Jie Yang

Xi'an Jiaotong University affiliated Northwest Women and Children's Hospital: Northwest Women and Children's Hospital

Ni Zhang

Xi'an Medical University

\section{Lei Wang}

Xi'an Jiaotong University affiliated Northwest Women and Children's Hospital: Northwest Women and Children's Hospital

$\mathrm{Na} \mathrm{Li}$

Xi'an Jiaotong University affiliated Northwest Women and Children's Hospital: Northwest Women and Children's Hospital

Juanzi Shi

Xi'an Jiaotong University affiliated Northwest Women and Children's Hospital: Northwest Women and Children's Hospital

Hanying Zhou ( $\sim 18709270206 @ 139 . c o m$ )

Hospital Group East: Groupement Hospitalier Est

\section{Research}

Keywords: frozen-thawed embryo transfer (FET), recurrent implantation failure (RIF), Gonadotropinreleasing hormone agonist, Hormone replacement cycle

Posted Date: November 23rd, 2021 
DOI: https://doi.org/10.21203/rs.3.rs-1090205/v1

License: (c) (1) This work is licensed under a Creative Commons Attribution 4.0 International License. Read Full License 


\section{Abstract}

\section{Background}

To determine whether Gonadotropin-releasing hormone $(\mathrm{GnRH})$ agonist down regulation combined with hormone replacement treatment (HRT) can improve the reproductive outcomes in the frozen thawed embryo transfer (FET) cycle of elderly patients with idiopathic recurrent implantation failure (RIF).

\section{Methods}

This is a retrospective cohort study analyzing 594 elderly patients (aged 36 43 years old) undergoing third cleavage embryo or blastocyst transfer over nearly 5-year period (January 2015-November 2020) at Northwest Women's and Children's Hospital after IVF-ICSI cycles. Patients with known endometriosis or adenomyosis were excluded from the study.According to different endometrial preparation protocols, patients were divided into three groups: natural cycle group $(N C, n=62)$, hormone replacement treatment cycle group (HRT, $n=194$ ) and $\mathrm{GnRH}$ agonist down regulation combined with HRT cycle group (GnRHaHRT, $n=290$ ). Live birth rate was primary outcome while clinical pregnancy rate, miscarriage rate, on going pregnancy rate were secondary outcomes.

\section{Results}

Live birth rate in the GnRHa-HRT group (31.90\%) was significantly higher than that in the HRT (21.65\%) or NC $(16.13 \%)$ groups $(P<0.0001)$. Logistic regression model adjusting for the potential confounders showed that patients in the GnRHa-HRT group have significantly higher live birth rate compared with those in the HRT group (OR, 2.708; 95\% Cl,1.251-5.864, $\mathrm{P}=0.011)$. However, live birth rate was not significantly different between GnRHa-HRT and NC groups (OR, 1.509; 95\% Cl,0.657-3.463, $\mathrm{P}=0.332$ ), which could be due to the small sample size in the NC group.

\section{Conclusions}

GnRHa-HRT protocol improves live birth rate in FET cycles of elderly patients with RIF. We hypothesize that GnRHa-HRT protocol enhances implantation related factors and promotes optimal endometrium receptivity, leading to the improved live birth rate. These findings are also useful for further investigating the underlying mechanism of GnRHa-HRT protocol in improving the reproductive outcomes of elderly patients with RIF.

Trial registration:The research protocol was approved by the hospital institutional ethics committee (2021002).

\section{Background}

Despite technical advances in in vitro fertilization (IVF), recurrent implantation failure (RIF), the absence of implantation after repeated embryo transfers, is still the biggest challenge of the current reproductive 
medicine. At present, there are a wide variety of options for the treatment of RIF, but none of the programs is effective for successful pregnancy.

Endometrium is the final destination of embryo implantation and successful implantation requires embryo interacting with a receptive endometrial lining. Endometrium and embryo interact and influence each other before embryo is transferred into the uterine cavity ${ }^{[1]}$. Therefore, both adequate endometrial receptivity and high embryonic quality are equally important for implantation. Prospective cohort studies have indicated that frozen-thawed embryo transfer (FET) may improve the reproductive outcomes in women with RIF ${ }^{[2]}$. In FET cycles, the embryos may be transferred into a more synchronous uterine environment, which can avoid the disturbed endometrial development due to ovarian stimulation.

It is currently still controversial with regard to which endometrial preparation protocol is more effective to promote implantation especially in the population of elderly women with RIF. A previous retrospective self-control study showed that GnRHa combined with hormone replacement therapy (GnRHa-HRT) protocol can increase the pregnancy success rate in FET cycles of patients who had experienced repeated failures of IVF treatment ${ }^{[3]}$. Other studies have further shown that the depot GnRHa protocol may protect the expression of endometrial receptivity marker and thereby improves endometrial receptivity ${ }^{[4-5]}$. Nevertheless, these findings have been questioned in a further retrospective cohort study showing that GnRHa-HRT protocol does not improve live birth rate for patients with RIF. However, patients of all ages were included in that study ${ }^{[6]}$.

Moreover, increasing maternal age as another risk of embryo-endometrium asynchrony makes the successful embryo implantation further complicated ${ }^{[7-8]}$. Yet, few studies have focused on reproductive outcomes in the elderly population with RIF. We hypothesized that before FET, elderly patients with idiopathic RIF might benefit from single-agent suppression with the use of $\mathrm{GnRH}$ agonist compared with those without $\mathrm{GnRH}$ agonist pretreatment.

\section{Methods}

\section{Study Design and Patients}

This study included elderly patients (aged 36-43 years old) undergoing FET cycle therapy at Northwest Women's and Children's Hospital after IVF/ICSI cycles between January 2015 and November 2020. This study only included patients undergoing the third or more embryo transfer after autologous IVF and ICSI (intracytoplasmic sperm injection) ( $n=594)$. Women who underwent embryo transfer after donor oocytes, women who were diagnosed with endometriosis, adenomyosis or both, and women whose endometrial thickness did not reach $7 \mathrm{~mm}$ on the day of transplantation were excluded. The research protocol was approved by the hospital institutional ethics committee (2021002).

\section{Endometrial preparation before frozen-thawed embryo transfer (FET)}


In the natural cycle (modified natural cycle FET) (NC group), we monitored when the leading follicles reached a mean diameter of $>17 \mathrm{~mm}$, human chorionic gonadotropin ( $\mathrm{hCG}, 10,000 \mathrm{IU})$ for triggering oocytes ovulation when serum luteinizing hormone $(\mathrm{LH})$ was $<20 \mathrm{IU} / \mathrm{L}$, or otherwise transvaginal ultrasound was performed every day until ovulation. FET was performed after 3 (cleavage embryo transfer) or 5 (blastocyst transfer) days.

In the HRT cycle (HRT group), starting at cycle day 5, estradiol valerate (Progynova; Bayer Schering Pharma AG, Berlin, Germany) was orally administrated daily. About 10 12 days later, $60 \mathrm{mg} / \mathrm{day}$ of natural progesterone in oil (progesterone; Xianju, Zhejiang, China) was injected i.m. to transform the endometrium as soon as the endometrial thickness reached $7 \mathrm{~mm}$ and serum $\mathrm{P}$ levels serum progesterone $(P)$ level was below $1.5 \mathrm{ng} / \mathrm{mL}$. Then, FET was performed after 4 (cleavage embryo transfer) or 6 (blastocyst transfer) days of progesterone therapy.

In the GnRH agonist down regulation combined with HRT cycle (GnRHa-HRT group), patients received single dose injection of $3.75 \mathrm{mg}$ long-acting triptorelin acetate on day 2 of the cycle after the ultrasound scan confirming ovarian quiescence and the presence of a thin endometrium ( $<5 \mathrm{~mm})$. After $28 \sim 30$ days, estrogen stimulation was initiated for HRT cycle without GnRH agonist.

\section{Luteal support}

Luteal support schemes included intramuscular injection of $60 \mathrm{mg} /$ day of natural progesterone in oil and oral uptake of $20 \mathrm{mg} /$ day progesterone in tablet (Dydrogesterone; Abbott Biologicals B.V., The Netherlands) daily ${ }^{[9-10]}$. After embryo transfer, all patients received luteal support until the day when plasma $\beta$-hCG concentration was determined (D14). If pregnancy was achieved, hormone administration was continued until 10 weeks of pregnancy.

\section{Outcome parameters}

The primary outcome parameter was live birth rate. The delivery of a viable infant after the 22nd gestational week was considered as the live birth. Secondary outcome parameters included clinical pregnancy rate (defined as the rate of an intrauterine gestational sac with fetal heart activity on ultrasound), ongoing pregnancy rate (defined as the rate of an intact pregnancy beyond at 12 weeks of gestation), miscarriagerate (the rate of a loss of clinical pregnancy before the 22 nd gestational week per clinical pregnancy cycle) ${ }^{[11]}$.

\section{Statistical Analysis}

Analysis of variance (ANOVA) was used for continuous variables (mean \pm standard deviation) and $x^{2}$ test was used for categorical variables to determine whether the distributions of covariates were statistically significant across treatment protocols. Multivariable logistic regression was used to identify the independent effect of treatment protocol on the live birth rate and miscarriagerate in patients with RIF, adjusting for patients' age, BMI, basal FSH level, infertility years, infertility type, endometrial thickness on transform day, number of embryos transferred and embryo transfer type. The results were manifested as 
odds ratio and $95 \%$ confidence interval $(\mathrm{Cl}) . \mathrm{P}<0.05$ was considered statistically significant. Data processing and statistical analysis were conducted using Statistical Package for Social science version 19.0 (SPSS, IBM, Inc.).

\section{Results}

A total of 594 women were enrolled into the study. The demographic and clinical characteristics of the patients were presented in Table 1. There were no significant differences in the age, BMI, basal FSH, infertility years and type of infertility among the three groups. The level of progesterone on the transform day in the GnRHa-HRT group was significantly lower than that in the HRT group ( $P=0.001)$. In addition, the number of embryos transferred and proportion of transferred blastocyst in the GnRHa-HRT group was significantly higher than those in the HRT group $(P=0.003$, and $P=0.044$, respectively). Furthermore, endometrial thickness on transform day was significantly different among the three groups $(P=0.002)$.

Table1 Baseline characteristics between different endometrial preparation groups 


\begin{tabular}{|c|c|c|c|c|}
\hline Characteristic & $\begin{array}{l}\text { GnRHa- } \\
\text { HRT } \\
(n=290)\end{array}$ & $\begin{array}{l}\text { HRT } \\
(n=194)\end{array}$ & $\begin{array}{l}\text { NC } \\
(n=62)\end{array}$ & $\begin{array}{l}\mathrm{P} \\
\text { value }\end{array}$ \\
\hline female age(y) & $38.69 \pm 2.19$ & $39.16 \pm 2.18$ & $38.73 \pm 2.00$ & 0.291 \\
\hline BMII (kg/m2) & $22.37 \pm 2.74$ & $22.72 \pm 2.76$ & $22.24 \pm 2.51$ & 0.647 \\
\hline bFSH(IU/L) & $7.95 \pm 3.04$ & $8.28 \pm 3.00$ & $7.99 \pm 3.16$ & 0.996 \\
\hline Infertility years(y) & $5.48 \pm 4.13$ & $5.23 \pm 4.26$ & $5.66 \pm 4.00$ & 0.768 \\
\hline Infertility type $(\mathrm{n}, \%)$ & & & & 0.407 \\
\hline Primary infertility & $76(26.21)$ & $46(23.71)$ & $20(32.26 \rrbracket$ & \\
\hline Secondary infertility & $214(73.79)$ & 148(76.29) & $42(67.74 \rrbracket$ & \\
\hline Fertilization type $(\mathrm{n}, \%)$ & & & & 0.065 \\
\hline IVF & $208(71.72)$ & 138(71.13) & $47(75.81)$ & \\
\hline ICSI & $78(2.69)$ & $53(27.32)$ & $11(17.74)$ & \\
\hline$|\mathrm{IVF}+| \mathrm{CSI}$ & $4(1.38)$ & $3(1.55)$ & $4(6.45)$ & \\
\hline Progesterone on the administration day $(\mathrm{ng} / \mathrm{ml})$ & $0.38 \pm 0.29$ & $0.53 \pm 0.47$ & & $0.001^{*}$ \\
\hline $\begin{array}{l}\text { Endometrial thickness on the day of } P \\
\text { administration }(\mathrm{mm})\end{array}$ & $10.36 \pm 1.69$ & $9.82 \pm 1.57$ & $10.83 \pm 2.26$ & $0.002^{*}$ \\
\hline No. of transferred embryos & $1.76 \pm 0.52$ & $1.69 \pm 0.55$ & $1.65 \pm 0.63$ & $0.003^{*}$ \\
\hline Type of embryo transferred(n, \%) & & & & $0.044^{*}$ \\
\hline Cleavage stage embryo & $131(45.17)$ & 109(56.19) & $34(54.84)$ & \\
\hline Blastocyst & $159(54.83)$ & $85(43.81)$ & $28(45.16)$ & \\
\hline
\end{tabular}

Values are means $\pm S D$ or number (percentage) of patients. ${ }^{\square} \mathrm{P}<0.05$ for $t$ test or chi-squared test;

Abbreviations: BMI body mass index; bFSH basal follicle-stimulating hormone; ICSI intracytoplasmic sperm injection; IVF in vitro fertilization

Clinical pregnancy rates, ongoing pregnancy rates and live birth rates in GnRHa-HRT group were significantly higher than those in the other two groups. However, the miscarriagerates did not differ significantly among the three groups (Table 2)

Table 2 FET treatment and pregnancy outcomes in the three groups 


\begin{tabular}{lllll} 
pregnancy outcomes & GnRHa-HRT & HRT & NC & P value \\
\hline Clinical pregnancy rate & $49.31(143 / 290)$ & $34.54(67 / 194)$ & $25.81(16 / 62)$ & $0.000^{*}$ \\
\hline On going pregnancy rate & $37.93(110 / 290)$ & $22.16(43 / 194)$ & $17.74(11 / 62)$ & $0.000^{*}$ \\
\hline Miscarriage rate & $22.38(32 / 143 \rrbracket$ & $35.82(24 / 67)$ & $31.25(5 / 16)$ & 0.114 \\
\hline Live birth rate & $36.90(107 / 290)$ & $21.65(42 / 194)$ & $16.13(10 / 62)$ & $0.000 *$
\end{tabular}

Values are percentage (no./no.) of patients. ${ }^{*} \mathrm{P}<0.05$ for $\mathrm{t}$ test or chi-squared test

Univariate analysis was performed to evaluate the effect of each variable on the reproductive outcomes (Table 3). The results showed that patients' age, number of embryos transferred, embryo transfer type and FET treatment protocol were associated with clinical pregnancy rate, ongoing pregnancy rate and live birth rate, while patients' age was the only variable associated with miscarriagerate.

Table 3 Single-factor analysis of factors related to FET outcomes 
Clinical indicators

Value

female age(y)

BMI

bFSH

Infertility years

Infertility type

Primary infertility

Secondary infertility

Endometrial Preparation Protocols

GnRHa-HRT

HRT

NC

Progesterone on the administration day

Endometrial thickness on the day of $\mathrm{P}$ administration

No. of transferred embryos

Type of embryo transferred

Cleavage stage embryo

Blastocyst

Clinical indicators

Value

Female age
Clinical pregnancy rate

OR value $(95 \%$

$\mathrm{Cl})$

$0.844(0.777-$

0.915)

$1.009(0.947-$

1.074)

$0.939(0.884-$

0.996)

0.965(0.925-

1.007)

Ref.

1.091(0.741-

1.606)
On going pregnancy rate OR value $(95 \% \quad P$ value $\mathrm{Cl}$ ) value value $\mathrm{Cl}$ ) value

0.000 * $0.785(0.715-\quad 0.000$ *
$0.861)$

0.788

1.008(0.9431.078)

$0.037 *$

0.936(0.8760.999)

0.098

0.959(0.9151.005)

Ref.

0.660

1.161(0.769-

1.753)
0.810

$0.047 *$

0.078
0.476

0.476
Ref.

2.797(1.514-

5.166)

1.517(0.799-

2.88)

0.585(0.333-

1.029)

1.088(0.986-

1.2)

1.563(1.136-

2.15)
Ref.

$0.001^{*}$

2.833(1.416-

5.668)

0.203

1.32(0.633-

2.752)

0.063

0.578(0.3111.076)

0.092

1.071(0.965-

1.188)

0.006 *

1.61(1.141-

2.27) 


\begin{tabular}{|c|c|c|c|c|}
\hline $\mathrm{BMI}$ & $\begin{array}{l}1.017(0.951- \\
1.088)\end{array}$ & 0.625 & $\begin{array}{l}0.992(0.891- \\
1.104)\end{array}$ & 0.880 \\
\hline bFSH & $\begin{array}{l}0.94(0.88- \\
1.004)\end{array}$ & 0.065 & $\begin{array}{l}1.023(0.922- \\
1.136)\end{array}$ & 0.668 \\
\hline Infertility years & $\begin{array}{l}0.958(0.914- \\
1.004)\end{array}$ & 0.076 & $\begin{array}{l}1.068(0.992- \\
1.149)\end{array}$ & 0.081 \\
\hline \multicolumn{5}{|l|}{ Infertility type } \\
\hline Primary infertility & Ref. & & Ref. & \\
\hline Secondary infertility & $\begin{array}{l}1.128(0.744- \\
1.711)\end{array}$ & 0.570 & $\begin{array}{l}0.897(0.456- \\
1.765)\end{array}$ & 0.752 \\
\hline \multicolumn{5}{|l|}{ Endometrial Preparation Protocols } \\
\hline GnRHa-HRT & Ref. & & Ref. & \\
\hline HRT & $\begin{array}{l}3.04(1.484- \\
6.231)\end{array}$ & $0.002^{*}$ & $\begin{array}{l}0.634(0.205- \\
1.959)\end{array}$ & 0.429 \\
\hline NC & $\begin{array}{l}1.437(0.673- \\
3.067)\end{array}$ & 0.349 & $\begin{array}{l}1.228(0.381- \\
3.953)\end{array}$ & 0.731 \\
\hline Progesterone on the administration day & $\begin{array}{l}0.582(0.311- \\
1.087)\end{array}$ & 0.090 & $\begin{array}{l}1.179(0.392- \\
3.545)\end{array}$ & 0.769 \\
\hline $\begin{array}{l}\text { Endometrial thickness on the day of } \mathrm{P} \\
\text { administration }\end{array}$ & $\begin{array}{l}1.096(0.987- \\
1.217)\end{array}$ & 0.085 & $\begin{array}{l}0.969(0.813- \\
1.154)\end{array}$ & 0.72 \\
\hline No. of transferred embryos & $\begin{array}{l}1.655(1.169- \\
2.343)\end{array}$ & $0.005^{\star}$ & $\begin{array}{l}0.788(0.435- \\
1.427)\end{array}$ & 0.432 \\
\hline \multicolumn{5}{|l|}{ Type of embryo transferred } \\
\hline Cleavage stage embryo & Ref. & & Ref. & \\
\hline Blastocyst & $\begin{array}{l}0.438(0.299- \\
0.641)\end{array}$ & $0.000^{*}$ & $\begin{array}{l}1.856(1.025- \\
3.364)\end{array}$ & $0.041 *$ \\
\hline
\end{tabular}

$* \mathrm{P}<0.05$ for Chi-square test

Abbreviations: OR odds ratio; $\mathrm{Cl} 95 \%$ confidence interval; $\mathrm{BMI}$ body mass index; $\mathrm{bFSH}$ basal folliclestimulating hormone;

In the adjusted model, GnRHa-HRT treatment protocol significantly increased the live birth rate compared with HRT treatment protocol $(\mathrm{OR}, 2.708 ; 95 \% \mathrm{Cl}, 1.251-5.864, \mathrm{P}=0.011$. However, the live birth rate was not significantly different between GnRHa-HRT and NC groups (Table 4).

Table 4 Multifactor analysis results of FET outcome-related factors 


\begin{tabular}{|lll|}
\hline Clinical indicators & Live birth rate & \\
\hline Value & OR value $(95 \% \mathrm{Cl})$ & P value \\
\hline Female age(y) & $0.815(0.735-0.904)$ & $0.000^{*}$ \\
\hline BMI & $0.998(0.927-1.075)$ & 0.963 \\
\hline bFSH & $0.965(0.897-1.037)$ & 0.333 \\
\hline Infertility years & $0.947(0.898-0.999)$ & $0.046^{*}$ \\
\hline Infertility type & & \\
\hline Primary infertility & Ref. & \\
\hline Secondary infertility & $1.084(0.661-1.779)$ & 0.748 \\
\hline Endometrial thickness on the day of P administration(mm) & $1.067(0.945-1.205)$ & 0.292 \\
\hline No. of transferred embryos & $2.017(1.341-3.032)$ & $0.001^{*}$ \\
\hline Type of embryo transferred & & \\
\hline Cleavage stage embryo & Ref. & \\
\hline Blastocyst & $0.456(0.290-0.717)$ & $0.001^{\star}$ \\
\hline Endometrial Preparation Protocols & & \\
\hline GnRHa-HRT & Ref. & $0.011^{*}$ \\
\hline HRT & $2.708(1.251-5.864)$ & \\
\hline NC & $1.509(0.657-3.463)$ & 0.332 \\
\hline
\end{tabular}

*P $<0.05$ for Chi-square test

Abbreviations: OR odds ratio; $\mathrm{Cl}$ 95\% confidence interval; BMI body mass index; bFSH basal folliclestimulating hormone;

\section{Discussion}

There was insufficient evidence to demonstrate that the use of depot $\mathrm{GnRHa}$ is better or worse than the $\mathrm{NC}$ or HRT protocol for the reproductive outcome in FET cycles, especially for patients with RIF.

Pretreatment with GnRHa before HRT in FET cycles is considered to be ineffective for the elderly patients, but it could be effective for the patients with RIF ${ }^{[12]}$. Recent studies also failed to demonstrate any significant effect of depot GnRHa-FET protocol in the live birth/ongoing pregnancy rate ${ }^{[6]}$. However, that study only included younger patients ( $₫ 40$ years) and thus the effect of depot GnRHa-FET protocol on elderly patients (36 43years) needs to be further evaluated. 
The present large-scale retrospective cohort study evaluated the reproductive outcomes in natural cycle, HRT cycle, GnRHa-HRT cycle of elderly patients with RIF when referred for FET. Our results showed that there was a significantly higher live birth rate in GnRHa-HRT treatment protocol than that in the NC and HRT protocols, while the miscarriagerate was not significantly different among the three protocols. In addition, we used low-dose drugs in our study. Patients in our study only received one intramuscular injection of $3.75 \mathrm{mg}$ depot leuprolide acetate. III-timed administration with the double dose of depot leuprolide acetate may increased not only the hypo-estrogenic side-effects, e.g., hot flushes,vaginal dryness, decreased libido and loss of bone density,but also high cost[13].

In contrast, previous retrospective self-control study suggested that GnRHa-HRT protocol, with 28 days of full-dose depot GnRHa, prolonged downregulation before ovarian stimulation, resulting in relatively high pregnancy success rates in idiopathic RIF patients undergoing IVF ${ }^{[3]}$.

Our results are consistent with previous studies showing that cryopreservation of all viable blastocysts after ICSI followed by a subsequent FET cycle can improve the implantation when compared with fresh $\mathrm{ET}^{[3,5,14-16]}$. Other studies showed that GnRHa may partially restore the endometrial secretion of implantation related factors, e.g., HOXA10, MEIS1 and LIF, which can regulate endometrial development and permit embryo implantation and decidualization ${ }^{[5,17-21]}$. Another study showed that ovarian stimulation with $\mathrm{GnRHa}$ partially restores the expression of endometrial integrin beta 3 and improves uterine receptivity in mice ${ }^{[22]}$.

An interesting observation in our study is that the mean level of progesterone on the administration day in the HRT cycle was significantly higher than that in the GnRHa-HRT cycles $(0.38 \pm 0.29$ versus $0.53 \pm 0.47$, $\mathrm{P}=0.001$ ), which is consistent with the results in previous studies ${ }^{[12,14,22]}$. This might be due to a premature rise of LH levels in hormonal stimulation protocol without a $\mathrm{GnRH}$ agonist, which negatively alters the receptive window of the endometrium ${ }^{[16]}$.

The NC protocol is one of the most frequently and widely used FET protocols in treatment cycles ${ }^{[24]}$. However, one previous study suggests that approximately two-thirds of implantation failures are due to disturbed endometrial receptivity and asynchronized embryo-endometrial cross-talk, while the quality of embryo is estimated to be responsible for the remaining one-third implantation failure ${ }^{[25]}$. However, in this study, we found that live birth rate in NC protocol was not significantly lower than that in the GnRHaHRT protocol, which may be due to the small sample size in the NC group. According to the histologic endometrial dating, the rate of a"displaced WOI (window of implantation)" in patients with RIF can reach $31.6 \%$, compared with $3.8 \%$ in the patients with good-prognosis $(P=0.003){ }^{[26]}$.

This is the first study to evaluate the optimal approach to prepare the endometrium in FET cycles of elderly patients with RIF. The limitation of this study is its retrospective design from a single medical center. However, through the analysis of large sample size using multivariate logistics model, some confounding factors were eliminated. In addition, the type of endometrial preparation was selected by the physician's preference and such preference may introduce a potential bias. Besides, we did not exclude 
cleavage stage of embryo transfer. This bias may be eliminated by a Cochrane review that blastocyst transfer over cleavage stage of embryo transfer was of low quality for live birth outcome ${ }^{\text {[27]. }}$

\section{Conclusions}

In conclusion, this nearly 5-year study provides very robust evidence regarding the reproductive potential of RIF women with increasing age. It showed that LBR in GnRHa-HRT protocol was raised compared with HRT protocol while the miscarriage rate was not affected. The live birth rate in NC protocol appears to be undamaged. However, the sample size of natural cycle protocol was too small, which may affect the outcome. Further multi-center randomized clinical trials are needed to confirm the possible benefits of GnRHa-HRT protocol for elderly patients with idiopathic recurrent implantation failure.

\section{Abbreviations}

FET: Frozen embryo transfer; HRT: Hormone replacement treatment; GnRHa-HRT: GnRH antagonist downregulation combined hormone replacement treatment; RIF: Repeated implantation failure; IVF:in vitro fertilization;ICSI:Intracytoplasmic sperm injection

\section{Declarations}

\section{Ethics approval and consent to participate}

This study was approved by the Ethics Committee of Northwest Women's and Children's Hospital and has been performed in accordance with the principles of Declaration of Helsinki.

\section{Consent for publication}

Not applicable.

\section{Availability of data and materials}

Not applicable.

\section{Competing interests}

The authors declare no competing interests.

\section{Funding}

\section{This work was supported by National Natural Science Foundation of China (82100083)}

\section{Authors' contributions}


DP, JY, LW,and NL designed the study, performed data analysis, and drafted the manuscript; NZ, $\mathrm{HZ}$ and $S Z$ critically review and corrected the final version of the manuscript. All participants approved the final version to be submitted.

\section{Acknowledgements}

The authors thank the staff from Northwest Women's and Children's Hospital for their assistance with the data collection. We thank all participants in this study.

\section{Authors' information}

1,The Assisted Reproduction Center, Northwest Women's and Children's Hospital, No. 73 Houzai Gate, Xincheng District, Xi'an City, Shaanxi Province, China

2 Department of Basic Medicine, Xi'an Medical University,Xin-Wang Street \#1, Xi'an 710021, Shaanxi, China

\section{References}

1. Margalioth EJ, Ben-Chetrit A, Gal M, Eldar-Geva T: Investigation and treatment of repeated implantation failure following IVF-ET. Hum Reprod 2006, 21:3036-3043.

2. Magdi Y, El-Damen A, Fathi AM, Abdelaziz AM, Abd-Elfatah Youssef M, Abd-Allah AA, Ahmed Elawady $M$, Ahmed Ibrahim M, Edris Y: Revisiting the management of recurrent implantation failure through freeze-all policy. Fertil Steril 2017, 108:72-77.

3. Yang $X$, Huang R, Wang YF, Liang XY: Pituitary suppression before frozen embryo transfer is beneficial for patients suffering from idiopathic repeated implantation failure. J Huazhong Univ Sci Technolog Med Sci 2016, 36:127-131.

4. Surrey ES, Minjarez DA, Schoolcraft WB: The incidence of aberrant endometrial alphavbeta(3) vitronectin expression in a high risk infertility population: could prolonged $\mathrm{GnRH}$ agonist therapy play a role? J Assist Reprod Genet 2007, 24:553-556.

5. Xu B, Geerts D, Hu S, Yue J, Li Z, Zhu G, Jin L: The depot GnRH agonist protocol improves the live birth rate per fresh embryo transfer cycle, but not the cumulative live birth rate in normal responders: a randomized controlled trial and molecular mechanism study. Hum Reprod 2020, 35:1306-1318.

6. Steiner N, Shrem G, Tannus S, Dahan SY, Balayla J, Volodarsky-Perel A, Tan SL, Dahan MH: Effect of GnRH agonist and letrozole treatment in women with recurrent implantation failure. Fertil Steril 2019, 112:98-104.

7. Ocal P, Cift T, Bulut B, Balcan E, Cepni I, Aydogan B, Irez T: Recurrent implantation failure is more frequently seen in female patients with poor prognosis. Int J Fertil Steril 2012, 6:71-78.

8. Shapiro BS, Daneshmand ST, Desai J, Garner FC, Aguirre M, Hudson C: The risk of embryoendometrium asynchrony increases with maternal age after ovarian stimulation and IVF. Reprod Biomed Online 2016, 33:50-55. 
9. Kim CH, Lee YJ, Lee KH, Kwon SK, Kim SH, Chae HD, Kang BM: The effect of luteal phase progesterone supplementation on natural frozen-thawed embryo transfer cycles. Obstet Gynecol Sci 2014, 57:291-296.

10. Mackens S, Santos-Ribeiro S, van de Vijver A, Racca A, Van Landuyt L, Tournaye H, Blockeel C: Frozen embryo transfer: a review on the optimal endometrial preparation and timing. Hum Reprod 2017, 32:2234-2242.

11. Zegers-Hochschild F, Adamson GD, Dyer S, Racowsky C, de Mouzon J, Sokol R, Rienzi L, Sunde A, Schmidt L, Cooke ID, et al: The International Glossary on Infertility and Fertility Care, 2017. Hum Reprod 2017, 32:1786-1801.

12. Dong M, Sun L, Huang L, Yi Y, Zhang X, Tan Y, Song G, Liu L, Wei F, Liu F: Gonadotropin-releasing hormone agonist combined with hormone replacement therapy does not improve the reproductive outcomes of frozen-thawed embryo transfer cycle in elderly patients: a retrospective study. Reprod Biol Endocrinol 2020, 18:73.

13. McLaren JS, Morris E, Rymer J: Gonadotrophin receptor hormone analogues in combination with add-back therapy: an update. Menopause Int 2012, 18:68-72.

14. El-Toukhy T, Taylor A, Khalaf Y, Al-Darazi K, Rowell P, Seed P, Braude P: Pituitary suppression in ultrasound-monitored frozen embryo replacement cycles. A randomised study. Hum Reprod 2004, 19:874-879.

15. Hill MJ, Miller KA, Frattarelli JL: A GnRH agonist and exogenous hormone stimulation protocol has a higher live-birth rate than a natural endogenous hormone protocol for frozen-thawed blastocyststage embryo transfer cycles: an analysis of 1391 cycles. Fertil Steril 2010, 93:416-422.

16. Ghobara T, Vandekerckhove P: Cycle regimens for frozen-thawed embryo transfer. Cochrane Database Syst Rev 2008:CD003414.

17. Satokata I, Benson G, Maas R: Sexually dimorphic sterility phenotypes in Hoxa10-deficient mice. Nature 1995, 374:460-463.

18. Taylor HS: The role of HOX genes in human implantation. Hum Reprod Update 2000, 6:75-79.

19. Du H, Taylor HS: The Role of Hox Genes in Female Reproductive Tract Development, Adult Function, and Fertility. Cold Spring Harb Perspect Med 2015, 6:a023002.

20. Hu L, Li H, Huang CL, Chen H, Zhu G, Qian K: Regulation of myeloid ecotropic viral integration site 1 and its expression in normal and abnormal endometrium. Fertil Steril 2014, 102:856-863 e852.

21. Aghajanova L: Update on the role of leukemia inhibitory factor in assisted reproduction. Curr Opin Obstet Gynecol 2010, 22:213-219.

22. Ruan HC, Zhu XM, Luo Q, Liu AX, Qian YL, Zhou CY, Jin F, Huang HF, Sheng JZ: Ovarian stimulation with $\mathrm{GnRH}$ agonist, but not GnRH antagonist, partially restores the expression of endometrial integrin beta3 and leukaemia-inhibitory factor and improves uterine receptivity in mice. Hum Reprod 2006, 21:2521-2529.

23. Johnson NP, Bagrie EM, Coomarasamy A, Bhattacharya S, Shelling AN, Jessop S, Farquhar C, Khan KS: Ovarian reserve tests for predicting fertility outcomes for assisted reproductive technology: the 
International Systematic Collaboration of Ovarian Reserve Evaluation protocol for a systematic review of ovarian reserve test accuracy. BJOG 2006, 113:1472-1480.

24. Groenewoud ER, Cantineau AE, Kollen BJ, Macklon NS, Cohlen BJ: What is the optimal means of preparing the endometrium in frozen-thawed embryo transfer cycles? A systematic review and metaanalysis. Hum Reprod Update 2017, 23:255-261.

25. Craciunas L, Gallos I, Chu J, Bourne T, Quenby S, Brosens JJ, Coomarasamy A: Conventional and modern markers of endometrial receptivity: a systematic review and meta-analysis. Hum Reprod Update 2019, 25:202-223.

26. Li Y, Li XF, Liao JN, Fan XX, Hu YB, Gan R, Lu G, Lin G, Gong F: Clinical value of histologic endometrial dating for personalized frozen-thawed embryo transfer in patients with repeated implantation failure in natural cycles. BMC Pregnancy Childbirth 2020, 20:527.

27. Glujovsky D, Farquhar C, Quinteiro Retamar AM, Alvarez Sedo CR, Blake D: Cleavage stage versus blastocyst stage embryo transfer in assisted reproductive technology. Cochrane Database Syst Rev 2016:CD002118. 